\title{
Development of a Novel Robot Design Strategy for Children with Autism to Improve Affective Touch Skill
}

\author{
Jaeryoung LEE \\ Graduate School of Engineering, Nagoya University
}

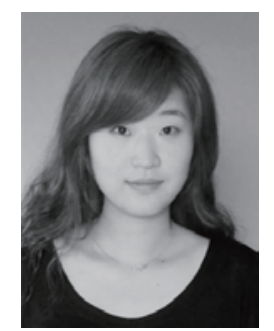

\section{Introduction}

Interactive robots have been developed as an effective tool for improvement of the social communication skills in children with autism. Recent studies show that the effectiveness of the human-robots interaction can be improved further if the robot provides proper feedback to the children when they demonstrate behaviour or social skills as expected. In this study, we explored the effect of colours on the interaction of the child with the robot.

\section{Method}

Four children diagnosed with low-functioning autism without other complications took part in the experiments. Participants were aged from 9 to 12 years (age: $M=10.75, S D=2.06$, gender: males). A special human machine interface (HMI) called Touch Ball was used for the experiments as shown in Fig. 1: (a) three axial force sensor, (b) the force sensor in centre of device, (c) transparent half sphere (d); the Touch Ball, used in the experiments.

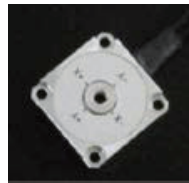

(a)

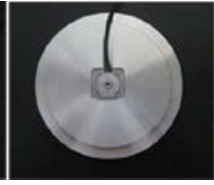

(b)

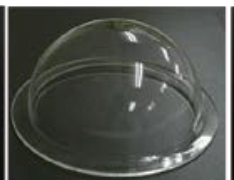

(c)

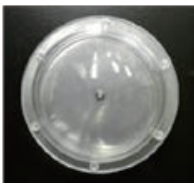

(d)
Fig. 1 Touch Ball

The experiments included three different scenarios; (I) Scenario with illuminated half-sphere, (II) scenario with changing colour of the circle on the computer screen, (III) scenario with verbal notification. In all three scenarios children needed to set their favourite colour by touching the Touch Ball and controlling the applied force until the favourite colour appeared.

Table 1 The relationships between force $\mathrm{F}$ and colour

\begin{tabular}{c||c|c|c}
\hline \hline $\mathrm{F}[\mathrm{mN}]$ & $\mathrm{F}<2$ & $2 \leq \mathrm{F}<3$ & $\mathrm{~F} \geq 3$ \\
\hline \hline Force & Weak force & Moderate force & Strong force \\
\hline Colour & Yellow & Red & Blue \\
\hline \hline
\end{tabular}

Depending on the value of force F, the colours changed. Four different colour circles (red, yellow, blue and green) were used in the experiment. A small humanoid robot, called as Palro was a part of each scenario to attract child's attention toward the experiment.

\section{Result}

We defined the target keeping time as the time for the favourite colour was kept: The experiments consisted of three sets with two trials and we allowed 20 seconds in each trial for the completion of the task. The success ratio was calculated from the target keeping rate that is the ratio between the target keeping time and the test time.

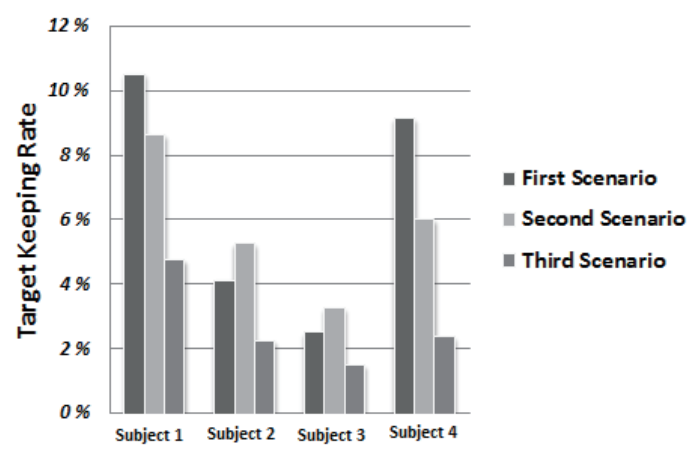

Fig. 2 The target keeping rate in three scenarios

The result achieved by two of the participants with autism, Subject 1 (age 9) and Subject 4 (age 12), showed that the target keeping rate of the first scenario was the highest; the results from the second scenario is lower than the first scenario. Other two autistic children (Subject 2 (age 9) and Subject 3 (age 11)) indicated slightly higher results on the second scenario comparing with first scenario. All participants showed the results from the third scenario were the lowest. The results in third scenario were matched our hypothesis that the results from the first scenario is higher than the third scenario, and also the result from the second scenario is higher than the third scenario. The experiments with colour feedback showed that the colour feedback can be understood easier by children with autism who are hyposensitive or hypersensitive. This study can contribute to better design of robot for autism therapy with improve autistic children's one of communication skill effectively. 